\title{
Dissecting the effects of Free Fatty Acids on the Thermodynamic Stability of Complex Model Membranes mimicking Insulin Secretory Granules
}

\author{
Francesca Saitta, Marco Signorelli and Dimitrios Fessas* \\ Dipartimento di Scienze per gli Alimenti, la Nutrizione e l'Ambiente, DeFENS, Università degli \\ Studi di Milano, Via Celoria 2, 20133, Milano, Italy \\ *Corresponding author. E-mail address: dimitrios.fessas@ unimi.it. Tel.: +390250319219
}

\begin{abstract}
:
A stepwise micro-DSC study of Small, Large and Giant Unilamellar Vesicles prepared as pure and mixed systems of DMPC, DPPC, DSPC and DOPC was performed, achieving the preparation of final model membranes whose phospholipid compositions represent the $75 \%$ in terms of the phospholipids tails and the $50 \%$ headgroups of the Insulin Secretory Granules (vesicles located in the pancreatic Langerhans $\beta$-cells and which are responsible for insulin and amylin storage and secretion in response to nutrient intake). Moreover, the effect of Free Fatty Acids, whose levels are recurrently altered in diabetic and/or obese subjects, on the thermodynamic stability of the final membranes was eventually investigated. The results allowed to discriminate each single thermodynamic contribution among the main factors that dictate the overall thermodynamic stability of these complex unilamellar systems evidencing mainly entropic effects hierarchically summarized as phospholipid unsaturations > phospholipid tail length > membrane curvature. The effect of the Free Fatty Acids highlighted a strong stabilizing effect on the membranes as well as more pronounced phase segregations in the case of saturated acids (palmitic and stearic), whereas the opposite effect was observed in the case of an unsaturated one (oleic).
\end{abstract}

Keywords: Model membranes, Differential Scanning Calorimetry, Free Fatty Acids, Insulin Secretory Granules. 


\section{Introduction}

Free Fatty Acids (FFAs) have been shown to be involved in several membrane-mediated cellular processes as membrane-bound enzyme activity [1], lipid-assisted protein transport across the bilayer [2], fusion of lipid vesicles and cells [3] and/or alteration of the microdomains of cell phospholipid bilayers as well as their physical properties [4,5]. Moreover, they also act as signalling molecules for several cell mechanisms [6,7], for instance insulin secretion [8]. Of course, the effect of FFAs on membranes and other cellular processes is dependent on their structural and chemical nature [9-12].

Several studies are reported to highlight the FFAs-membrane interaction mainly using spectroscopic, imaging, molecular dynamics and/or theoretical approaches [13-16], but, to our knowledge, only few works are devoted to a thermodynamic characterization of the role of the FFAs on the overall membrane stability. Moreover, most of these studies, including the calorimetric approach $[17,18]$, describe model systems whose, for instance, do not resemble the complexity of the composition of real biological vesicles [19]. Indeed, each modification in lipid composition and morphological characteristics might produce modifications of the molecular structure of such systems, which in turn might lead to variations of their physicochemical properties [20-22].

In this work we propose a calorimetric study of the influence of FFAs on the membrane thermodynamic stability $[11,23,24]$ discriminating compositional and morphological aspects by means of model membranes with progressive complexity aimed to simulate a real membrane, namely the phospholipid bilayer of the Insulin Secretory Granules (ISGs). ISGs are vesicles (average size of about $0.4 \mu \mathrm{m}$ ) located in the pancreatic Langerhans $\beta$-cells and which are responsible for insulin and amylin storage and secretion in response to nutrient intake [25]. Altered FFAs levels are recurrent in diabetic and/or obese subjects and plasma FFAs concentration is generally high in both with levels that tend to be increasingly higher with the increase of weight [26]. Specifically for Type 2 Diabetes Mellitus (T2DM), studies regarding the possible involvement of FFAs in the onset and/or progression of the disease are widely reported in the literature, referring both to their possible involvement in altered metabolic pathways [27,28] and to the direct action of FFAs on membranes [29]. Moreover, the action of FFAs has also been hypothesized to play a role in the interaction of amylin, an amyloidogenic protein, with cell membranes likely leading to the pancreatic $\beta$-cells failure by apoptosis [30].

In this frame, in order to preliminary discriminate the morphological and compositional effects on the overall membrane stability, we performed a thermodynamic study of different Small, Large and Giant Unilamellar Vesicles (SUVs, LUVs and GUVs) at physiological pH by using the microDSC technique. The phase behaviour of such vesicles prepared as pure and mixed system of 
DMPC, DPPC, DSPC and DOPC was studied, achieving the preparation of final model membranes whose phospholipids proportionally follow their abundancy [19] and represent the 75\% of the phospholipids' tails and the $50 \%$ of the headgroups in real ISGs [31]. Indeed, in order to discriminate the single thermodynamic contributions on some of the main parameters that dictate the thermodynamic stability of unilamellar membranes, namely vesicle size, tails length, presence of unsaturations and components' molar ratio, for this paper all the constituents were chosen with the same headgroup (choline). The effect of three different FFAs, such as palmitic, stearic and oleic acids, added in different amounts to the final systems was eventually investigated highlighting a hierarchy of the interactions involved.

\section{Materials and methods}

\subsection{Materials}

1,2-dimyristoyl-sn-glycero-3-phosphocholine (DMPC), 1,2-dipalmitoyl-sn-glycero-3phosphocholine (DPPC), 1,2-distearoyl-sn-glycero-3-phosphocholine (DSPC) and 1,2-dioleoyl-snglycero-3-phosphocholine (DOPC) powders were purchased from Avanti Polar Lipids (purity certified by the supplier $>99 \%$ ), whereas palmitic acid (PA), stearic acid (SA) and oleic acid (OA), as well as the other chemicals, were obtained from Sigma Aldrich. The lipids were of the highest available purity $(\geq 99 \%)$ and were used without further purification. All solvents were of analytical grade.

\subsection{Liposomes preparation}

\subsubsection{Preparation of Giant Unilamellar Vesicles (GUVs)}

GUVs were prepared following a procedure described elsewhere [32]. Briefly, the phospholipids were dissolved in chloroform obtaining a $2 \mathrm{mM}$ concentration. A volume equal to $1 \mathrm{~mL}$ of this solution was transferred in a round-bottom flask where, subsequently, $7 \mathrm{~mL}$ of $10 \mathrm{mM}$ phosphate buffer ( $\mathrm{pH}$ 7.4) were added. The organic solvent was removed through rotary evaporation (Heidolph Laborota 4000 efficient, WB eco, Schwabach, Germany) under reduced pression at $40^{\circ} \mathrm{C}$ and $40 \mathrm{rpm}$, obtaining about $6.0 \mathrm{~mL}$ of GUVs dispersion at about $0.3 \mathrm{mM}$ lipid concentration.

\subsubsection{Preparation of Large Unilamellar Vesicles (LUVs)}

Large vesicles were obtained from GUVs dispersions, which were extruded through polycarbonate filters (pore size of 400nm) mounted on a heated mini-extruder (Avanti Polar Lipids, 
Alabaster, AL, USA) fitted with two 1mL gastight syringes (Hamilton, Reno, NV, USA). The extrusion from already unilamellar giant vesicles assured us the unilamellarity of the large ones (supplementary material). An odd number of passages, usually 41, was performed to avoid any contamination by liposomes that might have not passed through the filters [33].

\subsubsection{Preparation of Small Unilamellar Vesicles (SUVs) and Free Fatty Acids (FFAs) addition}

Liposomes were prepared through thin-film hydration [34]. An amount of about 20mg phospholipids was dissolved in $2 \mathrm{~mL}$ of chloroform. The solvent was removed through rotary evaporation (Heidolph Laborota 4000 efficient, WB eco, Schwabach, Germany) at $40^{\circ} \mathrm{C}$ and the films were first kept under vacuum for at least 3 hours to remove solvent traces and then aged overnight at $4^{\circ} \mathrm{C}$. For the hydration, $10 \mathrm{mM}$ phosphate buffer $(\mathrm{pH} \mathrm{7.4)}$ ) at a temperature above the gel-to-liquid-crystal transition of the lipid system was added up to a $10 \mathrm{mg}$ lipid/mL concentration. The obtained mixture was vortexed and slowly stirred in water bath, at the same temperature chosen for the buffer, for about an hour until the induction of a homogenous emulsion. Extrusion was performed through $100 \mathrm{~nm}$ polycarbonate filters following the same precautions previously described.

As for FFAs-containing membranes, the acids were mixed with phospholipids prior to dissolve them in chloroform.

\subsubsection{Preparation of Multilamellar Lipid Bilayers (MLBs)}

MLBs samples were prepared by simply dispersing the adequate lipid powder amounts in $10 \mathrm{mM}$ phosphate buffer achieving a $0.2 \mathrm{M}$ lipid concentration.

\subsection{Spectroscopic characterization}

The hydrodynamic diameter of all liposomal formulations was measured by Dynamic Light Scattering (DLS). Amounts of $100 \mu \mathrm{L}$ of SUVs preparations and $670 \mu \mathrm{L}$ of LUVs and GUVs preparations were diluted in buffer up to $3 \mathrm{~mL}$ after the annealing of the dispersions. Measurements were performed at $25^{\circ} \mathrm{C}$ through a light-scattering instrument (Litesizer ${ }^{\mathrm{TM}} 500$, Anton Paar, Graz, Austria) in side-scatter mode for SUVs and LUVs dispersion, whereas the back-scatter mode was used for GUVs dispersions. The results permitted to verify the specifications about the polycarbonate filters' pore sizes used for the SUVs and LUVs extrusions [33] and to verify that the GUVs sizes was higher than $1 \mu \mathrm{m}$ (supplementary material).

\subsection{Thermal analysis measurements}


Calorimetry was used to determine the stability of the membranes with specific reference to the transitions of the lipid phases. Micro-DSC was selected as the most suitable technique for liposome investigation [23]. The instrument used was a Setaram micro DSCIII (Setaram Instrumentation, Caluire, France) operating with $1 \mathrm{~mL}$ hermetically closed pans at $0.5^{\circ} \mathrm{C} / \mathrm{min}$ scanning rate. After the conclusion of the liposomes' preparation protocols, each dispersion was allowed to anneal for at least $30 \mathrm{~min}$ at room temperature before launching the DSC measurement. SUVs samples were diluted up to $2.5 \mathrm{mM}$ phospholipids concentration, also for vesicles which included FFAs, whereas GUVs and LUVs suspensions were used without further dilutions $(\sim 0.3 \mathrm{mM})$. The final phospholipid concentration for all kind of vesicles was checked by using the Stewart assay [35].

Instead, classic DSC technique was selected to obtain thermograms for Multilamellar Lipid Bilayers (MLBs) as references. A PerkinElmer DSC6 (PerkinElmer, Waltham, MA, USA) working with hermetically closed pans was used by choosing a $0.5^{\circ} \mathrm{C} / \mathrm{min}$ scanning rate. About $20 \mathrm{mg}$ of samples with $0.2 \mathrm{M}$ lipid concentration were loaded in the pans for each run.

The raw data were worked out with the dedicated software "THESEUS" [36]. Briefly, the apparent specific heat trace, $C_{p}{ }^{a p p}(T)$, was scaled to obtain the excess specific heat, $C_{p}{ }^{e x c}(T)$, with respect to the low temperature lipids state. Due to such a treatment, the area beneath the recorded peaks directly corresponded to the relevant transitions enthalpy $\Delta H^{\circ}$ of the lipid phase. Two heating-cooling cycles were applied to each sample. All transitions were reversible and the second cycle heating curves were considered to evaluate the parameters of the thermotropic transitions observed. Errors were evaluated on the basis of at least three replicas.

In case of single main peaks, the main transition temperature, $T_{m}$, is usually identified as the temperature of the calorimetric peak maximum, $T_{\max }$, and the transition cooperativity is quantified by means of $\Delta T_{1 / 2}$ (full width at half maximum) that is of the order of $0.5^{\circ} \mathrm{C}$ for MLBs, while the unilamellar liposomes' values are generally 2-3 times higher according to the literature [23]. However, in this work we are often dealing with complex signals. In order to describe the overall stability of the systems, in addition to $T_{\max }$ we propose the use of the transition average temperature, $\bar{T}$, defined here as

$$
\bar{T}=\int_{T_{0}}^{T_{f}} T \cdot f(T) d T
$$

being $T_{0}$ and $T_{f}$, the initial and final limit of the observable peak and where the frequency function $f(T)$ is just the normalized calorimetric peak distribution

$$
f(T)=\frac{C_{p}^{e x c}(T)}{\Delta H^{\circ}}
$$


Furthermore, to better estimate the cooperativity in multicomponent and/or multiphasic samples, we propose an Average Cooperativity Index, $A C I$, defined here as

$$
A C I=\sqrt{\int_{T_{0}}^{T_{f}}(T-\bar{T})^{2} \cdot f(T) d T} .
$$

This approach is just a classical statistical distribution approach applied to the calorimetric signal (for instance, ACI coincides with the standard deviation $\sigma$ in the case of a gaussian distribution) and just for comparison we mention that in case of single peaks, as for the MLBs, a $\Delta T_{1 / 2}$ value of 0.5 ${ }^{\circ} \mathrm{C}$ corresponds to $\sim 0.2{ }^{\circ} \mathrm{C}$ in ACI terms, whereas the $\left|\bar{T}-T_{\text {max }}\right|$ difference represents a peak asymmetry index.

\section{Results and discussion}

\subsection{One-component systems}

The DSC thermograms for DMPC, DPPC and DSPC samples as MLBs, GUVs, LUVs and SUVs are shown in Fig. 1 (detailed figures are reported in supplementary material).

Fig1.approximately.here

In particular, the comparison between the thermograms of one-component systems as Multilamellar Bilayers (MLBs) and Unilamellar Vesicles is shown in Fig. 1a (LUVs are reported as an example). We observed that the phenomena involved were totally reversible and the cooling traces evidenced hysteresis effects typical of "Ising" systems [37]. As regards the MLBs, we observed the distinctive sharp signal related to the main transition from the gel phase to the liquid crystal phase, preceded by the typical smaller and broader pretransition [23]. Moreover, the temperature ranges covered by these signals and the corresponding main transition enthalpies followed the trend outlined by the phospholipids' chain lengths increment (14, 16, 18 carbon atoms for DMPC, DPPC and DSPC respectively) [38,39]. The Unilamellar Vesicles followed the same trend as regards the temperature ranges but, in this case, the signals were evidently much broader in line with the literature [23]. Indeed, it is well known that the cooperativity of the phase transition depends on the geometry of the membrane and decreases as the vesicle curvature increases $[23,40]$. Such low cooperativity reflects a dispersion of lipid regions (with different stability and cooperativity parameters) that might be further affected by the presence of perturbing agents.

In any case, the main transition enthalpies remained correspondingly unaffected indicating that such curvature effects were mostly of entropic nature in line with previous studies [23,39-42]. 
We may note here that, according to the literature [43], the curvature effects to the overall membrane stability for one-component systems seems are enhanced in vesicles smaller than 70nm, despite in some cases discrepancies emerges, especially as for the enthalpic contribution [44]. However, these systems are not solutions but suspensions and experimental conditions, in some cases, may play a role in the DSC profiles [23]. Here, the measures of one-component systems were performed as reference for direct comparison with the more complex systems' micro-DSC curves produced at the same experimental conditions.

In summary, for all the forms of the systems, as overall main transition values we obtained $\Delta H^{\circ}=$ $(44 \pm 2) \mathrm{kJ} \cdot \mathrm{mol}^{-1}$ for DSPC,$\Delta H^{\circ}=(35 \pm 2) \mathrm{kJ} \cdot \mathrm{mol}^{-1}$ for DPPC, and $\Delta H^{\circ}=(24 \pm 2) \mathrm{kJ} \cdot \mathrm{mol}^{-1}$ for DMPC. The pretransition was better detected in the case of MLBs $\left(\Delta H^{\circ}=(7 \pm 1) \mathrm{kJ} \cdot \mathrm{mol}^{-1}, \Delta H^{\circ}=\right.$ $(5 \pm 1) \mathrm{kJ} \cdot \mathrm{mol}^{-1}$ and $\Delta H^{\circ}=(4 \pm 1) \mathrm{kJ} \cdot \mathrm{mol}^{-1}$ for DSPC, DPPC and DMPC, respectively) whereas they became less evident going from GUVs, LUVs to SUVs [45,46].

As regards the variations in $T_{\max }$ among different bilayer curvatures, we remind here that, in the case of the extruded vesicles, we are in presence of large asymmetric DSC profiles and the $T_{\max }$ of the curve is an apparent quantity and may not be directly correlated with a $T_{m}$ corresponding to an "all-in-one" gel-to-liquid crystalline transition. In any case, the differences of $T_{\max }$ among different bilayer curvatures for the same one-component system were in the narrow range of $\pm 0.2^{\circ} \mathrm{C}$ (including experimental error) with respect to the average $T_{m}$ between the four bilayer forms for the same system. In summary, for all the forms of the systems, we obtained $T_{\max }=(54.9 \pm 0.2){ }^{\circ} \mathrm{C}$ for DSPC and $T_{\max }=(41.6 \pm 0.2){ }^{\circ} \mathrm{C}$ for DPPC and $T_{\max }=(24.0 \pm 0.2){ }^{\circ} \mathrm{C}$ for DMPC.

The micro-DSC thermograms for the same pure systems as GUVs and SUVs are reported in Fig. $1 \mathrm{~b}$ in order to better evidence the curvature entropic effects between pure unilamellar systems. As expected [40], the higher the curvature (SUVs), the lower the cooperativity. In summary, the ACI values (see material and methods) we obtained were of the same order for the DSPC, DPPC and DMPC systems, namely $\sim 0.2 \pm 0.1{ }^{\circ} \mathrm{C}$ for MLBs, $\sim 0.6 \pm 0.1$ for GUVs, $\sim 1.0 \pm 0.1$ for LUVs and $\sim 1.2 \pm 0.1$ for SUVs.

However, despite these differences are still appreciable comparing unilamellar vesicles with different sizes in the cases of one-component systems, different composition ratios for more complex membranes might influence such a behaviour. Indeed, our main purpose was to explore the thermodynamic behaviour of different and more complex model cell membranes that are as close as possible to reality against some perturbing agents, specifically in this case the ISGs against FFAs in the frame of T2DM. For this reason, a careful evaluation from a hierarchical point of view of any possible phenomenon that might influence the thermodynamic stability of real cell membranes is essential. 


\subsection{Binary systems}

In order to evaluate the contribution of each phospholipid on the thermodynamic stability of a hypothetical model membrane, preliminary experiments on binary systems prepared as 1:1 molar mixtures of DSPC:DPPC, DPPC:DMPC and DSPC:DMPC were performed for the size border systems, i.e. GUVs and SUVs (Fig. 2). The relevant thermodynamic data are reported in Table 1.

Fig2.and.Table1.approximately.here

At a first glance, for phospholipids mixtures with a chain lengths' deviation of two carbon units, i.e. DSPC:DPPC and DPPC:DMPC, we observed a main peak roughly placed in the middle of the temperature range defined by the $T_{\max }$ values of the one-component dispersions (considered as references), in line with the literature $[38,47]$. Such profiles indicate that phospholipids with slight differences in tails' length are in first approximation miscible, i.e. almost thermodynamically compatible. However, we may notice broader signals than the references' profiles, also presenting slight shoulders towards the phospholipids with the shortest tails.

Conversely, in the case of DSPC:DMPC mixture, i.e. phospholipids with a higher chain lengths' deviation (four carbon units), these effects were dramatically enhanced showing a complex asymmetric and mainly biphasic signal. Consequently, we may argue that the presence of phase separations, i.e. the degree of thermodynamic incompatibility, is strictly related to the lipid composition in terms of chain lengths' deviation [48]. However, we remind that this conclusion regards saturated phospholipids with the same headgroup.

Notwithstanding, the thermograms showed the formation of phases characterized by different lipid molar ratios for all the three mixtures. Indeed, a small step at the beginning of the peaks may be noticed in all the cases evidencing the presence of phases richer in the lower- $T_{m}$ phospholipid (corresponding to DPPC-rich phase in DSPC:DPPC and to DMPC-rich phase in both DSPC:DMPC and DPPC:DMPC). However, regardless of the magnitude of the phase separations characterizing such systems, we observed that the lipids reorganization within the vesicles promoted the enrichment of the most stable domains and such a detail clearly emerges by the asymmetry of the peaks in Fig. 2.

The overall enthalpies obtained for the three binary mixtures are collected in Table 1 and are compared with the respective enthalpy simply arithmetically calculated adding the enthalpy values obtained for the main transition in the case of one-component systems considering the composition ratio [49]. We observed higher $\Delta H^{\circ}$ than the calculated values for all the systems with deviations of 
about $4-5 \mathrm{~kJ} \cdot \mathrm{mol}^{-1}$ that are of the order of the pretransition values. Accordingly, we may argue that these differences may reflect the contribution of residual pretransitions of each constituent not clearly detectable because covered by the overall curves. In any case, no considerable enthalpic differences are evident between GUVs and SUVs.

As regards the effects ascribable to the curvature for vesicles with binary composition (Fig. 2), the differences between GUVs and SUVs become almost negligible for the DSPC:DPPC mixture, whereas they are still visible in the cases of mixtures with the shortest phospholipid DMPC. This behaviour when DMPC is one of the component, above all in the case of DPPC:DMPC, may be related to the higher gap between the $T_{\max }$ values of the respective reference systems if compared to the value associated to the DSPC:DPPC mixture (for instance, $\Delta T_{\max }$ is about $13.3^{\circ} \mathrm{C}$ for DSPC:DPPC, whereas it is about $17.6^{\circ} \mathrm{C}$ for DPPC:DMPC). However, it is less evident in the case of DSPC:DMPC because of the breadth of the profiles. In any case, the differences observed, due to the membrane curvature, are less evident if compared to the one-component systems reported in Fig. 1b. Nevertheless, despite we do not exclude that local curvature modifications may be involved in some functionality in real membranes [20,21], it has to be underlined that the results on binary systems shown in Fig.2 regard DMPC:DPPC systems prepared as 1:1 molar ratio whereas only 1:7 correspond in the ISGs [31]. Consequently, these curvature effects should be smaller in the real system and may be considered of minor importance in this study with respect to the composition contribution to the overall membrane stability and further exploitation of these fine curvature aspects is beyond the scope of this work. A tentative interpretation on the curvature effects depletion trend is that in pure systems the order-disorder differences due to the asymmetry between the inner and outer leaflets of the bilayer are more evident because the starting point (let's say the GUVs suspension) is more ordered. In binary systems the starting point is an already perturbed structure with respect the one-component systems and this may decrease the order-disorder difference with the respective SUVs.

\subsection{Model membranes and FFAs influence}

Fig3.approximately.here

Fig. 3 reports the micro-DSC traces of a completely saturated ternary membrane (7 DPPC : 2 DSPC : 1 DMPC) and a quaternary membrane which included the $5 \%$ of an unsaturated component (DOPC) in the ternary membrane (6.7 DPPC : 1.8 DSPC : 1.0 DMPC : 0.5 DOPC), both prepared as SUVs dispersions. As indicated above, these compositions reflect the components proportions 
and represent the $50 \%$ of the headgroups and an average of $75 \%$ of the tails in real ISGs [31]. We remind that in this work we considered only one type of headgroup (choline) in order to focus on the influence of the phospholipid tails in terms of length and/or unsaturation, reflecting the phospholipid ratio of the real membrane.

As for the completely saturated (ternary) membrane, we observed a thermogram that resembles the profile exhibited by the binary systems in Fig. 2. Indeed, we observed a main peak with a slightly higher maximum temperature $\left(42.8^{\circ} \mathrm{C}\right)$ than the $T_{\max }$ of the major component (DPPC), which reflects the presence of a little amount of DSPC, as well as the small "step" at the beginning of the curve, which reflects the presence of DMPC. Indeed, the temperature range of the initial part of the curve corresponds with the DPPC:DMPC mixed phases reported in Fig. 2. Conversely, in the case of the quaternary membrane the thermogram appeared asymmetric and broader, showing a more homogeneous phases distribution as a consequence of the addition of unsaturations fully covering the initial step of the ternary membrane's profile. Moreover, the $T_{\max }$ is slightly shifted towards lower temperatures $\left(41.7^{\circ} \mathrm{C}\right)$.

The enthalpies observed are reported in Table 1 and are in line with those calculated by simple arithmetic addition of the references' enthalpies following the components proportions. The comparison between the experimental and the calculated enthalpies indicated that the pretransitions are prevented in such complex systems and once again confirmed the entropic nature of the mixing phenomena that influence the thermodynamic stability of these membranes. On the other hand, the introduction of an unsaturated component has a stronger influence (considering the small amount added) as regards both the phases distribution that become broader and more homogenous and the entropic effect that compromise the overall thermodynamic stability. Such effects are often related to the concept of "membrane flexibility" that is well known to play an important role in the biological frame [23,50]. All these effects allow to tentatively depict a scenario describing a hierarchy of the observed entropic contributions on the thermodynamic stability of these model membranes, i.e. presence of unsaturations $>$ tails length $>$ curvature.

The effects of the FFAs on the thermal stability of such complex systems are shown in Fig.4 and the relevant thermodynamic parameters are reported in Table 1.

\section{Fig4.approximately.here}

At a first view, we observed that the saturated FFAs produced strong stabilizing effects in both membranes, whereas opposite effects were exhibited in the presence of the unsaturated one. 
As regards the enthalpic contribution to the overall thermodynamic stability, in the case of saturated FFAs as palmitic and stearic acids we observed a slight enthalpy increase (Table 1) that seemed to reach a saturation level, with the exception of palmitic acid in the ternary membrane for which the enthalpic increment followed the FFAs amount added and became relevant.

Taking into account that the gel-to-liquid crystalline phase transitions observed are of "orderdisorder" type and assuming that the disordered state (liquid crystalline phase) is not strongly affected by the presence of perturbing molecules, the transition variations should depend mainly on variations of the ordered state. Consequently, we may argue that the interaction between FFAs and membrane phospholipids enhances the number of tail-tail contacts in the ordered state (gel phase) that is revealed in an increase of transition enthalpy. The enthalpy saturation effects observed for SA and not for PA suggests that the saturated free acids preferably interact with phospholipids with the same tail length. Indeed, considering the molar composition of the membrane, which is rich in DPPC (70\%), the PA (whose tail is as long as the DPPC ones) enthalpy effects were more relevant. On the other hand, the presence of an unsaturated FFA as the oleic acid caused opposite effects indicating that the presence of an unsaturated chain between the phospholipid tails disturbs the gel phase and produces a decrease of the overall transition enthalpy in both membranes.

As for the entropy contribution, the presence of saturated FFAs caused a strong entropic stabilizing effect shifting the curves at higher temperatures and an evident amplification of phase separations that may be identified in at least two main domains in both the ternary and quaternary membranes. The phase separations observed again suggests preferable interactions that enhanced the ordered state of the membrane, according to the aliphatic chains length differences as already observed in the binary systems. Indeed, the DSC profiles reported in Fig. 4 and the ACI values (Table 1) suggest that these entropic effects are dependent on the FFAs length, becoming stronger as the length increases (for instance SA in our case). The overall effect depended on the FFA amount added and seemed to enrich the population of the more stable domain.

Conversely, the presence of the oleic acid caused a destabilizing effect, in line with the enthalpy decrement, lowering the transition temperature region and broadening the peaks again.

As for the differences between the ternary and quaternary membranes, i.e. the presence of $5 \%$ of DOPC that leads to more "perturbed" bilayers, the enthalpic effects were attenuated in all cases and their contribution in the overall stabilization remained modest regardless of the FFAs amount, assigning again a predominant role to the entropic effects that dictate the thermodynamic stability. The overall picture of the entropic differences between the two membranes is presented in Fig. 5 in terms of shift of the transition average temperature, $\bar{T}$, of such systems with respect to the pure membranes values. 
Fig5.approximately.here

According to this comparison, we may conclude that in first approximation the main effects are common to these membranes. In particular, in the case of saturated FFAs the stabilizing effects depend on the number of carbon units (chain's length) and are more pronounced in less stable membranes, for instance the quaternary one. On the contrary, in the case of unsaturated FFAs the destabilizing effects are more pronounced in more stable membranes, such as the ternary one.

In any case, we observed an overall loss of transition cooperativity (Table 1) and the effects were dependent of the amount of FFAs included, i.e. no saturation of such mainly entropic effects were observed in the concentration range investigated.

\section{Conclusions}

The experimental evidences of this work, which followed a stepwise approach as regard the membranes complexity keeping constant the influence of the phospholipids headgroup, permitted to discriminate the basic effects that dictate the membrane thermodynamic stability and the influence of the FFAs inclusion. We may conclude that the entropic differences between MLBs and Unilamellar Vesicles are important in terms of cooperativity (being maximum in MLBs) and the systems are difficult to compare, in line with the literature [23]. Curvature effects are also relevant among unilamellar vesicles of different size in pure systems, but become of minor importance in more complex systems, also just including binary composition membranes. The overall stability of the mixed membranes depends on the relative molar ratio of the saturated phospholipids (the longer the tails, the more stable the membrane) and the constituent's contribution is somehow "additive". Moreover, the presence of phase separations increases as the tail lengths' gap between the phospholipid components increases. On the other hand, the presence of unsaturated phospholipids, even in minor amounts, has a relevant overall destabilizing effect and enhance the homogeneousness on the phases distribution. All the mentioned effects are mainly of entropic nature, while the enthalpic contributions seem just proportional to the single component contributions according to the composition.

As for the influence of some perturbing agents, the presence of saturated FFAs on both the membranes that are representative of the ISGs $(50 \%$ of the headgroups and an average of $75 \%$ of the tails) produces a mainly entropic strong stabilizing effect but also an enthalpic contribution and induces severe phase separations in any case. On the opposite, the unsaturated FFA produces an 
overall destabilization of the membranes. All these main effects depend on the amount of FFAs present. Whether these thermodynamic effects are correlated with physiological aspects of the real ISGs, local or general, is beyond the scope of this paper. Indeed, as a first stage in this paper the phospholipid headgroup (choline) was chosen the same for all the constituents. Furthermore, the absence in the formulation of an essential component such as cholesterol, that strongly influences the membranes stability, further decreases the representative of these model membranes with the real system. The effect of cholesterol, the influence of different headgroups and how such differences influence the generality of the conclusions as regard both the membrane thermodynamic stability and the FFAs influence in real membranes will be the subject of a future work. However, despite these necessary limitations as first step, the systematic dissecting of the single thermodynamic contributions to the overall thermodynamic behaviour of the ISGs obtained in this work may be useful also as a general view since the main composition of many biological membranes consists in the phospholipid components here investigated more or less with similar composition ratios $[19,51]$.

\section{Acknowledgments}

The Authors are grateful to prof. Costas Demetzos and dr. Natassa Pippa (Dept. of Pharmaceutical Technology, National and Kapodistrian University of Athens, Greece) for the fruitful discussions as regard the membrane assembly and prof. Luciano Piergiovanni (DeFENS, University of Milan, Italy) for the comments as regard the light scattering characterization.

\section{References}

[1] G. Schmalzing, P. Kutschera, Modulation of ATPase activities of human erythrocyte membranes by free fatty acids or phospholipase A2, J. Membr. Biol. 69 (1982) 65-76. doi:10.1007/BF01871243.

[2] C. La Rosa, S. Scalisi, F. Lolicato, M. Pannuzzo, A. Raudino, Lipid-assisted protein transport: A diffusion-reaction model supported by kinetic experiments and molecular dynamics simulations, J. Chem. Phys. 144 (2016) 184901. doi:10.1063/1.4948323.

[3] C.E. Creutz, cis-Unsaturated fatty acids induce the fusion of chromaffin granules aggregated by synexin, J. Cell Biol. 91 (1981) 247-256. doi:10.1083/jcb.91.1.247.

[4] R.D. Klausner, A.M. Kleinfeld, R.L. Hoover, M.J. Karnovsky, Lipid domains in membranes. Evidence derived from structural perturbations induced by free fatty acids and lifetime 
heterogeneity analysis., J. Biol. Chem. 255 (1980) 1286-1295.

[5] M. Ibarguren, D.J. López, P. V. Escribá, The effect of natural and synthetic fatty acids on membrane structure, microdomain organization, cellular functions and human health, Biochim. Biophys. Acta - Biomembr. $1838 \quad$ (2014) 1518-1528. doi:10.1016/j.bbamem.2013.12.021.

[6] A.R. Brash, Arachidonic acid as a bioactive molecule, J. Clin. Invest. 107 (2001) 1339-1345. doi:10.1172/JCI13210.

[7] A.P. Desbois, V.J. Smith, Antibacterial free fatty acids: activities, mechanisms of action and biotechnological potential, Appl. Microbiol. Biotechnol. 85 (2010) 1629-1642. doi:10.1007/s00253-009-2355-3.

[8] Y. Itoh, Y. Kawamata, M. Harada, M. Kobayashi, R. Fujii, S. Fukusumi, K. Ogi, M. Hosoya, Y. Tanaka, H. Uejima, H. Tanaka, M. Maruyama, R. Satoh, S. Okubo, H. Kizawa, H. Komatsu, F. Matsumura, Y. Noguchi, T. Shinohara, S. Hinuma, Y. Fujisawa, M. Fujino, Free fatty acids regulate insulin secretion from pancreatic $\beta$ cells through GPR40, Nature. 422 (2003) 173-176. doi:10.1038/nature01478.

[9] S.S. Funari, F. Barceló, P. V. Escribá, Effects of oleic acid and its congeners, elaidic and stearic acids, on the structural properties of phosphatidylethanolamine membranes, J. Lipid Res. 44 (2003) 567-575. doi:10.1194/j1r.M200356-JLR200.

[10] J. Davidsen, O.G. Mouritsen, K. Jørgensen, Synergistic permeability enhancing effect of lysophospholipids and fatty acids on lipid membranes, Biochim. Biophys. Acta - Biomembr. 1564 (2002) 256-262. doi:10.1016/S0005-2736(02)00461-3.

[11] P. Høyrup, J. Davidsen, K. Jørgensen, Lipid Membrane Partitioning of Lysolipids and Fatty Acids: Effects of Membrane Phase Structure and Detergent Chain Length, J. Phys. Chem. B. 105 (2001) 2649-2657. doi:10.1021/jp003631o.

[12] R. Koynova, B. Tenchov, Interactions of surfactants and fatty acids with lipids, Curr. Opin. Colloid Interface Sci. 6 (2001) 277-286. doi:10.1016/S1359-0294(01)00094-2.

[13] J. Kurniawan, K. Suga, T.L. Kuhl, Interaction forces and membrane charge tunability: Oleic acid containing membranes in different $\mathrm{pH}$ conditions, Biochim. Biophys. Acta - Biomembr. 1859 (2017) 211-217. doi:10.1016/j.bbamem.2016.11.001.

[14] J. Prades, S.S. Funari, P. V. Escribá, F. Barceló, Effects of unsaturated fatty acids and triacylglycerols on phosphatidylethanolamine membrane structure, J. Lipid Res. 44 (2003) 1720-1727. doi:10.1194/jlr.M300092-JLR200.

[15] I. Zavodnik, A. Zaborowski, A. Niekurzak, M. Bryszewska, Effect of free fatty acids on erythrocyte morphology and membrane fluidity, IUBMB Life. 42 (1997) 123-133. 
doi:10.1080/15216549700202501.

[16] H. Jespersen, J.H. Andersen, H.J. Ditzel, O.G. Mouritsen, Lipids, curvature stress, and the action of lipid prodrugs: Free fatty acids and lysolipid enhancement of drug transport across liposomal membranes, Biochimie. 94 (2012) 2-10. doi:10.1016/j.biochi.2011.07.029.

[17] A. Ortiz, J.C. Gómez-Fernández, A differential scanning calorimetry study of the interaction of free fatty acids with phospholipid membranes, Chem. Phys. Lipids. 45 (1987) 75-91. doi:10.1016/0009-3084(87)90041-7.

[18] S.E. Schullery, T.A. Seder, D.A. Weinstein, D.A. Bryant, Differential thermal analysis of dipalmitoylphosphatidylcholine-fatty acid mixtures, Biochemistry. 20 (1981) 6818-6824. doi:10.1021/bi00527a012.

[19] J.E. Rothman, J. Lenard, Membrane Asymmetry, Science (80-. ). 195 (1977) 743-753.

[20] A. Tonnesen, S.M. Christensen, V. Tkach, D. Stamou, Geometrical Membrane Curvature as an Allosteric Regulator of Membrane Protein Structure and Function, Biophys. J. 106 (2014) 201-209. doi:10.1016/j.bpj.2013.11.023.

[21] R. Parthasarathy, J.T. Groves, Curvature and spatial organization in biological membranes, Soft Matter. 3 (2007) 24-33. doi:10.1039/B608631D.

[22] H.T. McMahon, J.L. Gallop, Membrane curvature and mechanisms of dynamic cell membrane remodelling, Nature. 438 (2005) 590-596. doi:10.1038/nature04396.

[23] K. Gardikis, S. Hatziantoniou, M. Signorelli, M. Pusceddu, M. Micha-Screttas, A. Schiraldi, C. Demetzos, D. Fessas, Thermodynamic and structural characterization of LiposomalLocked in-Dendrimers as drug carriers, Colloids Surfaces B Biointerfaces. 81 (2010) 11-19. doi:10.1016/j.colsurfb.2010.06.010.

[24] S.R. Shaikh, D.S. LoCascio, S.P. Soni, S.R. Wassall, W. Stillwell, Oleic- and docosahexaenoic acid-containing phosphatidylethanolamines differentially phase separate from sphingomyelin, Biochim. Biophys. Acta - Biomembr. 1788 (2009) 2421-2426. doi:10.1016/j.bbamem.2009.08.019.

[25] A. Young, Tissue Expression and Secretion of Amylin, in: Adv. Pharmacol., 2005: pp. 1945. doi:10.1016/S1054-3589(05)52002-7.

[26] P. Björntorp, H. Bergman, E. Varnauskas, Plasma free fatty acid turnover rate in obesity, Acta Med. Scand. 185 (2009) 351-356. doi:10.1111/j.0954-6820.1969.tb07347.x.

[27] W. El-Assaad, J. Buteau, M.-L. Peyot, C. Nolan, R. Roduit, S. Hardy, E. Joly, G. Dbaibo, L. Rosenberg, M. Prentki, Saturated Fatty Acids Synergize with Elevated Glucose to Cause Pancreatic $\beta$-Cell Death, Endocrinology. 144 (2003) 4154-4163. doi:10.1210/en.2003-0410.

[28] K. Maedler, G.A. Spinas, D. Dyntar, W. Moritz, N. Kaiser, M.Y. Donath, Distinct Effects of 
Saturated and Monounsaturated Fatty Acids on $\beta$-Cell Turnover and Function, Diabetes. 50 (2001) 69-76. doi:10.2337/diabetes.50.1.69.

[29] D. Milardi, M.F.M. Sciacca, L. Randazzo, A. Raudino, C. La Rosa, The Role of Calcium, Lipid Membranes and Islet Amyloid Polypeptide in the Onset of Type 2 Diabetes: Innocent Bystanders or Partners in a Crime?, Front. Endocrinol. (Lausanne). 5 (2014) 1-4. doi:10.3389/fendo.2014.00216.

[30] P. Westermark, A. Andersson, G.T. Westermark, Islet Amyloid Polypeptide, Islet Amyloid, and Diabetes Mellitus, Physiol. Rev. 91 (2011) 795-826. doi:10.1152/physrev.00042.2009.

[31] M.J. MacDonald, L. Ade, J.M. Ntambi, I.-U.H. Ansari, S.W. Stoker, Characterization of Phospholipids in Insulin Secretory Granules and Mitochondria in Pancreatic Beta Cells and Their Changes with Glucose Stimulation, J. Biol. Chem. 290 (2015) 11075-11092. doi:10.1074/jbc.M114.628420.

[32] A. Moscho, O. Orwar, D.T. Chiu, B.P. Modi, R.N. Zare, Rapid preparation of giant unilamellar vesicles., Proc. Natl. Acad. Sci. 93 (1996) 11443-11447. doi:10.1073/pnas.93.21.11443.

[33] R.C. MacDonald, R.I. MacDonald, B.P.M. Menco, K. Takeshita, N.K. Subbarao, L. Hu, Small-volume extrusion apparatus for preparation of large, unilamellar vesicles, Biochim. Biophys. Acta - Biomembr. 1061 (1991) 297-303. doi:10.1016/0005-2736(91)90295-J.

[34] A. Laouini, C. Jaafar-Maalej, I. Limayem-Blouza, S. Sfar, C. Charcosset, H. Fessi, Preparation, Characterization and Applications of Liposomes: State of the Art, J. Colloid Sci. Biotechnol. 1 (2012) 147-168. doi:10.1166/jcsb.2012.1020.

[35] J.C.M. Stewart, Colorimetric determination of phospholipids with ammonium ferrothiocyanate, Anal. Biochem. 104 (1980) 10-14. doi:10.1016/0003-2697(80)90269-9.

[36] G. Barone, P. Del Vecchio, D. Fessas, C. Giancola, G. Graziano, THESEUS: A new software package for the handling and analysis of thermal denaturation data of biological macromolecules, J. Therm. Anal. 38 (1992) 2779-2790. doi:10.1007/BF01979752.

[37] E. Ising, "Report on the theory of ferromagnetism." Beitrag zur Theorie des Ferromagnetismus, (1924) 253-258.

[38] S. Mabrey, J.M. Sturtevant, Investigation of phase transitions of lipids and lipid mixtures by sensitivity differential scanning calorimetry., Proc. Natl. Acad. Sci. 73 (1976) 3862-3866. doi:10.1073/pnas.73.11.3862.

[39] R. Koynova, M. Caffrey, Phases and phase transitions of the phosphatidylcholines, Biochim. Biophys. Acta - Rev. Biomembr. 1376 (1998) 91-145. doi:10.1016/S0304-4157(98)00006-9.

[40] V.P. Ivanova, T. Heimburg, Histogram method to obtain heat capacities in lipid monolayers, 
curved bilayers, and membranes containing peptides, Phys. Rev. E. 63 (2001) 041914. doi:10.1103/PhysRevE.63.041914.

[41] H. Yokoyama, K. Ikeda, M. Wakabayashi, Y. Ishihama, M. Nakano, Effects of Lipid Membrane Curvature on Lipid Packing State Evaluated by Isothermal Titration Calorimetry, Langmuir. 29 (2013) 857-860. doi:10.1021/la304532k.

[42] A. Jutila, P.K.J. Kinnunen, Novel Features of the Main Transition of Dimyristoylphosphocholine Bilayers Revealed by Fluorescence Spectroscopy, J. Phys. Chem. B. 101 (1997) 7635-7640. doi:10.1021/jp971400k.

[43] D. Lichtenberg, E. Freire, C.F. Schmidt, Y. Barenholz, P.L. Felgner, T.E. Thompson, Effect of surface curvature on stability, thermodynamic behavior, and osmotic activity of dipalmitoylphosphatidylcholine single lamellar vesicles, Biochemistry. 20 (1981) 34623467. doi:10.1021/bi00515a024.

[44] J. Drazenovic, H. Wang, K. Roth, J. Zhang, S. Ahmed, Y. Chen, G. Bothun, S.L. Wunder, Effect of lamellarity and size on calorimetric phase transitions in single component phosphatidylcholine vesicles, Biochim. Biophys. Acta - Biomembr. 1848 (2015) 532-543. doi:10.1016/j.bbamem.2014.10.003.

[45] T. Heimburg, A Model for the Lipid Pretransition: Coupling of Ripple Formation with the Chain-Melting Transition, Biophys. J. 78 (2000) 1154-1165. doi:10.1016/S00063495(00)76673-2.

[46] T. Heimburg, Mechanical aspects of membrane thermodynamics. Estimation of the mechanical properties of lipid membranes close to the chain melting transition from calorimetry, Biochim. Biophys. Acta - Biomembr. 1415 (1998) 147-162. doi:10.1016/S0005-2736(98)00189-8.

[47] P. Losada-Pérez, N. Mertens, B. de Medio-Vasconcelos, E. Slenders, J. Leys, M. Peeters, B. van Grinsven, J. Gruber, C. Glorieux, H. Pfeiffer, P. Wagner, J. Thoen, Phase Transitions of Binary Lipid Mixtures: A Combined Study by Adiabatic Scanning Calorimetry and Quartz Crystal Microbalance with Dissipation Monitoring, Adv. Condens. Matter Phys. 2015 (2015) 1-14. doi:10.1155/2015/479318.

[48] L.A. Bagatolli, E. Gratton, A Correlation between Lipid Domain Shape and Binary Phospholipid Mixture Composition in Free Standing Bilayers: A Two-Photon Fluorescence Microscopy Study, Biophys. J. 79 (2000) 434-447. doi:10.1016/S0006-3495(00)76305-3.

[49] P.W.M. Van Dijck, A.J. Kaper, H.A.J. Oonk, J. De Gier, Miscibility properties of binary phosphatidylcholine mixtures. A calorimetric study, Biochim. Biophys. Acta - Biomembr. 470 (1977) 58-69. doi:10.1016/0005-2736(77)90061-X. 
[50] K.N. Kontogiannopoulos, A. Dasargyri, M.F. Ottaviani, M. Cangiotti, D. Fessas, V.P. Papageorgiou, A.N. Assimopoulou, Advanced Drug Delivery Nanosystems for Shikonin: A Calorimetric and Electron Paramagnetic Resonance Study, Langmuir. (2018). doi:10.1021/acs.langmuir.8b00751.

[51] S. Ramanadham, F.-F. Hsu, S. Zhang, A. Bohrer, Z. Ma, J. Turk, Electrospray ionization mass spectrometric analyses of phospholipids from INS-1 insulinoma cells: comparison to pancreatic islets and effects of fatty acid supplementation on phospholipid composition and insulin secretion, Biochim. Biophys. Acta - Mol. Cell Biol. Lipids. 1484 (2000) 251-266. doi:10.1016/S1388-1981(00)00022-6. 


\section{Figure captions}

\section{Fig. 1}

DSC thermograms for pure DMPC, DPPC and DSPC dispersions (from left to right) representing a) MLBs (thin curves) versus LUVs (bold curves) and b) GUVs (thin curves) versus SUVs (solid curves). The dotted vertical lines mark the $T_{m}$ of the respective MLBs.

\section{Fig. 2}

Micro-DSC thermograms for GUVs (thin curves) and SUVs (solid curves) dispersions obtained by 1:1 DSPC:DPPC, DPPC:DMPC and DSPC:DMPC mixtures. The dotted vertical arrows mark the $T_{m}$ of pure DMPC, DPPC, and DSPC MLBs (left to right).

\section{Fig. 3}

Micro-DSC thermograms for a completely saturated ternary membrane as 7 DPPC : 2 DSPC : 1 DMPC molar ratio (solid curve) and a quaternary membrane obtained by the addition of the $5 \%$ of DOPC to the ternary membrane achieving a 6.7 DPPC : 1.8 DSPC : 1.0 DMPC : 0.5 DOPC molar ratio (dashed curve). The dotted vertical arrow marks the $T_{m}$ of pure DPPC MLBs.

\section{Fig. 4}

Micro-DSC profiles for pure vesicles (solid curves) and vesicles with the addition of the $10 \%$ (dashed curves) and the 25\% (dotted curves) of FFAs. Thermograms are reported for the saturated ternary membrane including a) palmitic acid, b) stearic acid and c) oleic acid, and for the quaternary membrane including d) palmitic acid, e) stearic acid and f) oleic acid.

\section{Fig. 5}

Histogram representation showing the effects of $10 \%$ and $25 \%$ of palmitic acid (PA), stearic acid (SA) and oleic acid (OA) on ternary (full bar) and quaternary (lined bar) model membranes in terms of transition average temperature $\bar{T}$. 


\section{Table 1.}

Thermodynamic parameters evaluated from micro-DSC investigations for 1) binary systems and 2) complex model membranes, namely main transition enthalpy $\left(\Delta H^{\circ}\right)$, peak maximum temperature $\left(T_{\text {max }}\right)$, transition average temperature $(\bar{T})$ and Average Cooperativity Index (ACI). The enthalpy arithmetical values calculated adding the reference values obtained from one-component systems according the composition ratio is also reported for comparison.

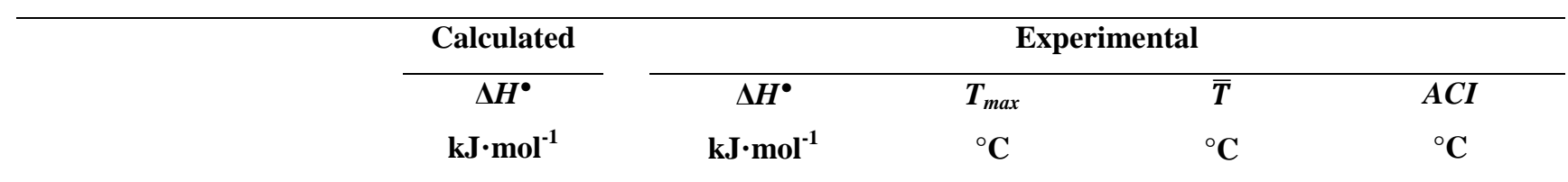

\section{Binary systems}

DSPC:DPPC

\begin{tabular}{|c|c|c|c|c|c|}
\hline GUVs & 40 & $46 \pm 2$ & $48.1 \pm 0.3$ & $47.3 \pm 0.3$ & $2.3 \pm 0.2$ \\
\hline SUVs & & $45 \pm 2$ & $48.4 \pm 0.3$ & $47.2 \pm 0.3$ & $2.0 \pm 0.2$ \\
\hline
\end{tabular}

DPPC:DMPC

GUVs

SUVs

30

DSPC:DMPC

GUVs

\section{Complex model membranes}

Pure Ternary Membrane

$$
\begin{aligned}
& +10 \% \mathrm{PA} \\
& +25 \% \mathrm{PA} \\
& +10 \% \mathrm{SA} \\
& +25 \% \mathrm{SA} \\
& +10 \% \mathrm{OA} \\
& +25 \% \mathrm{OA}
\end{aligned}
$$

Pure Quaternary Membrane

$+10 \%$ PA

$+25 \%$ PA

$+10 \% \mathrm{SA}$

$+25 \% \mathrm{SA}$

$+10 \%$ OA

$+25 \%$ OA
36

32

$37 \pm$

$42 \pm 2$

$55 \pm 2$

$41 \pm 2$

$41 \pm 2$

$30 \pm 2$

$30 \pm 2$

$33 \pm 2$

$38 \pm 2$

$41 \pm 2$

$39 \pm 2$

$38 \pm 2$

$33 \pm 2$

$34 \pm 2$
$41.7 \pm 0.3$

$41.7 \pm 0.3$

$45.0 \pm 0.3$

$44.8 \pm 0.3$

$37.4 \pm 0.3$
$37.1 \pm 0.3$

$7.1 \pm 0.2$

$6.9 \pm 0.2$

$3.0 \pm 0.2$

$3.3 \pm 0.2$

$31.9 \pm 0.3$

$6.9 \pm 0.2$ 



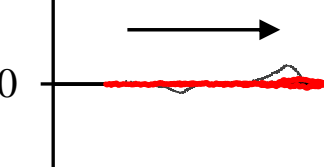

$-20$ $U^{2}$ $-40$

$-60$

$-80$

$-100$

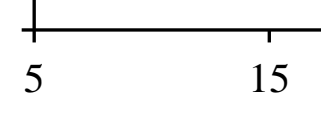

b

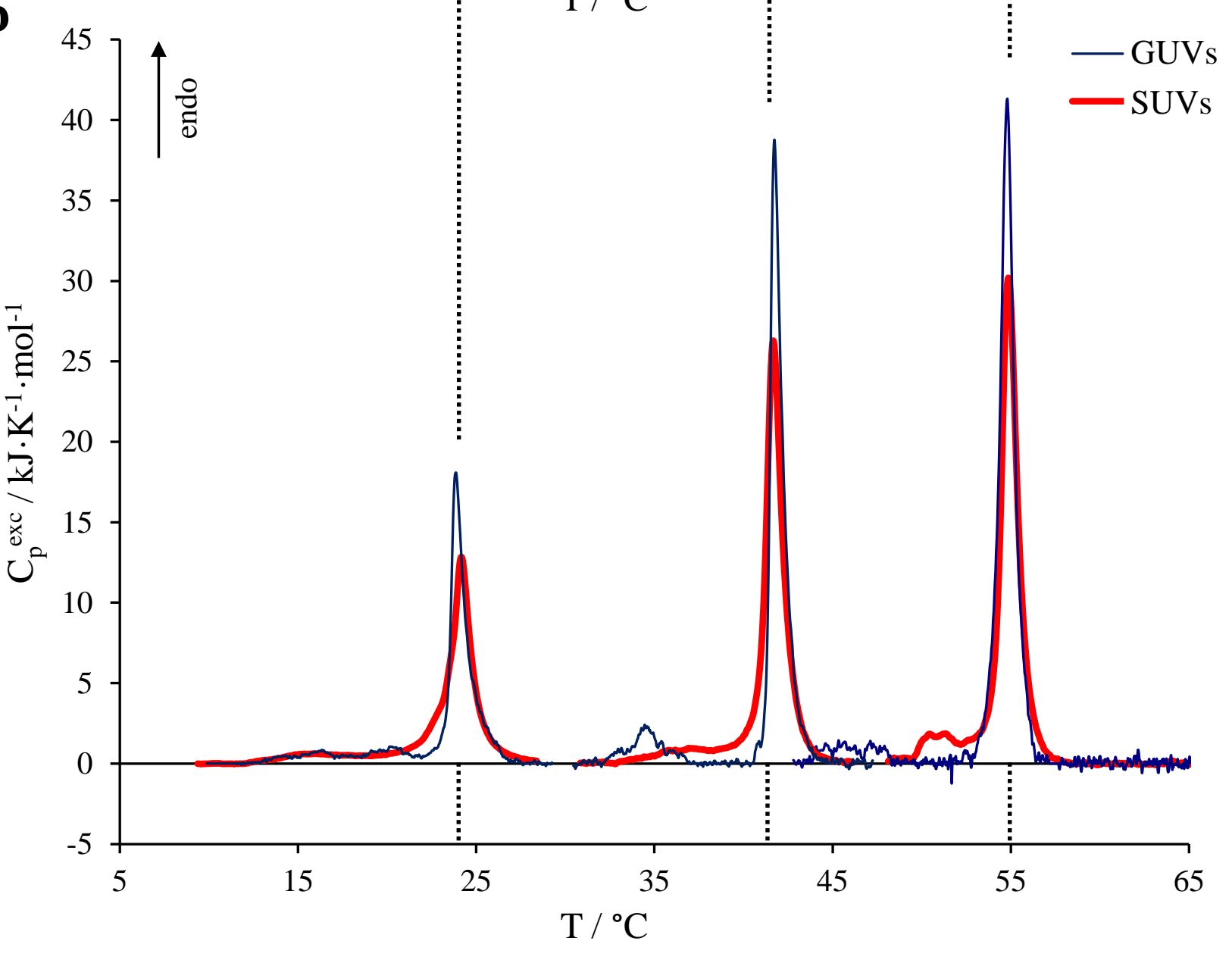




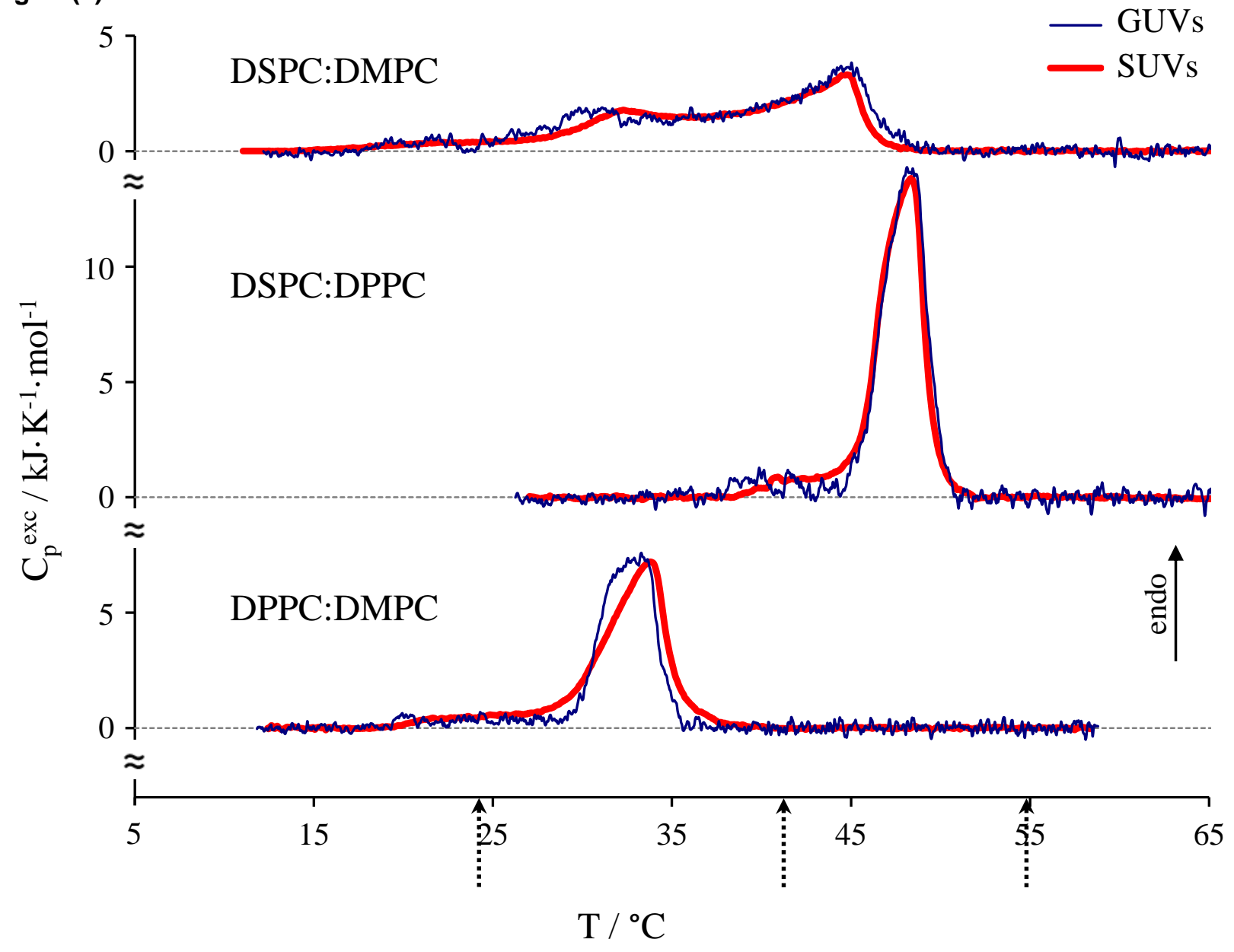


Figure(s)

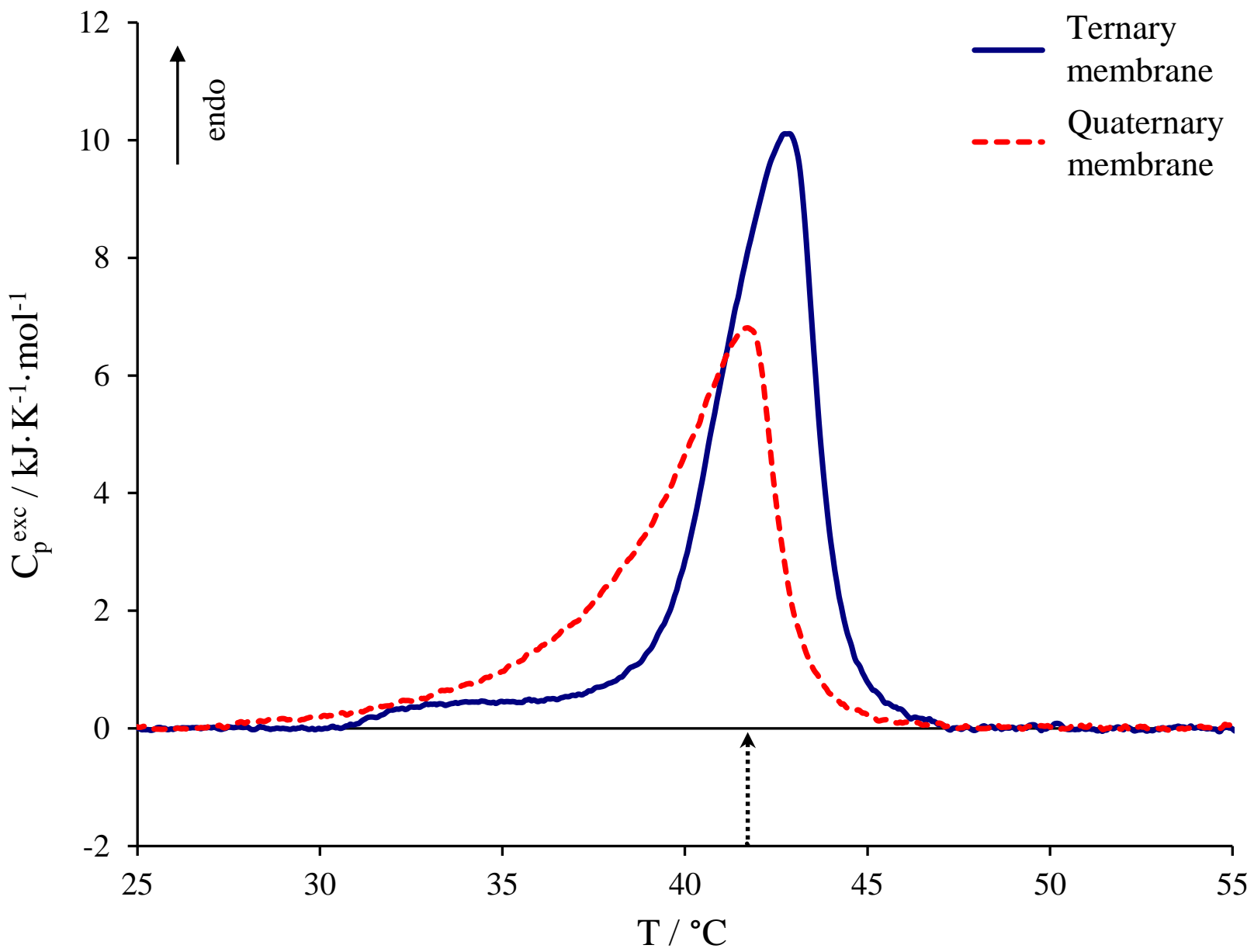




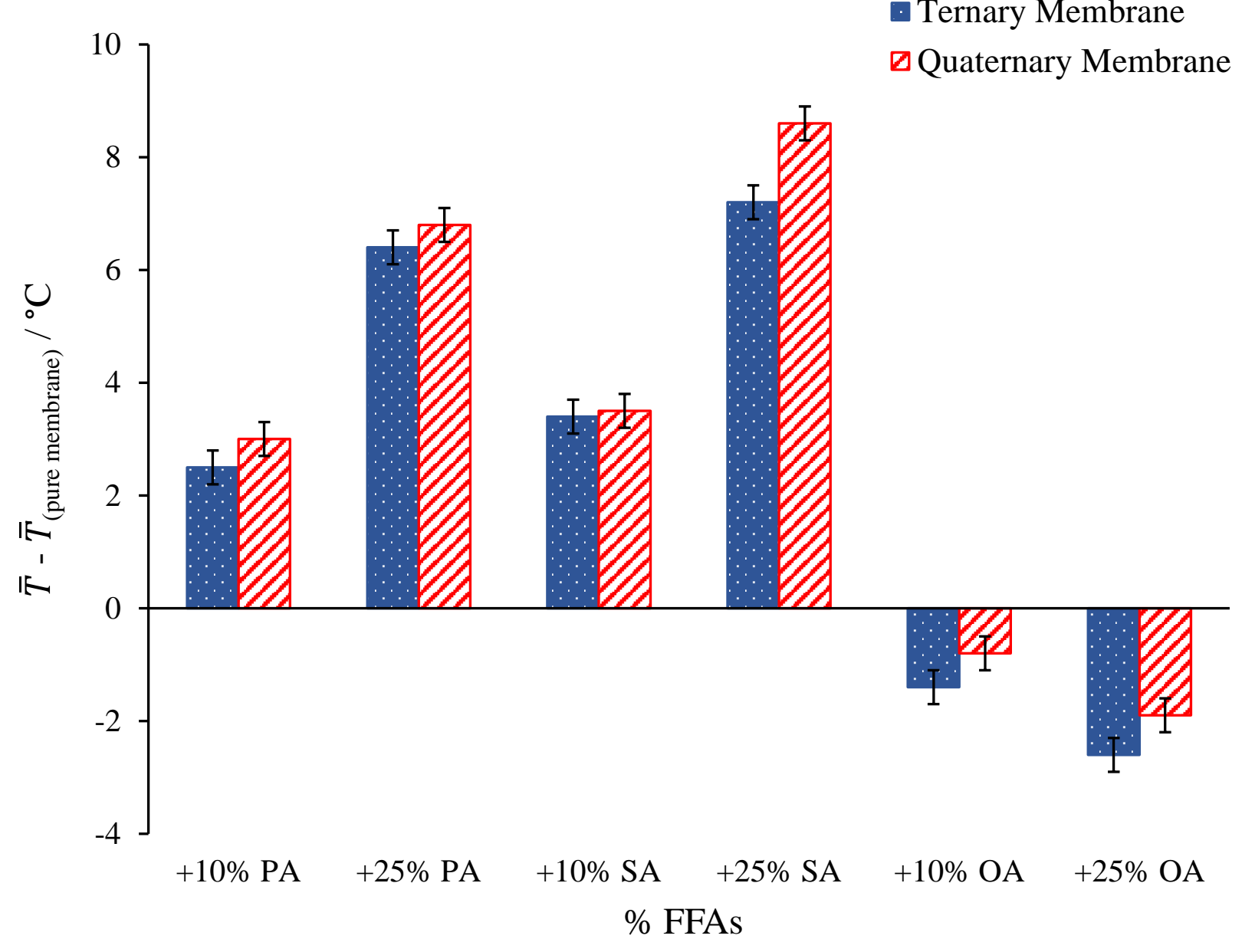


Supplementary Material
Click here to download Supplementary Material: SupplementaryMaterialFessasREV.pdf

Supplementary Material
Click here to download Supplementary Material: SupplementaryMaterialFessasREV.pdf

Click here to download Supplementary Material: SupplementaryMaterialFessasheV.pdf

(n)

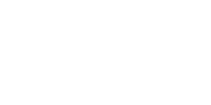

(1)

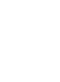

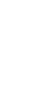
(1) (1)

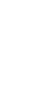

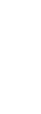
.

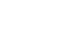

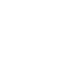

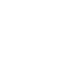

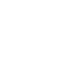

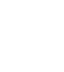

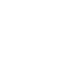

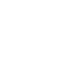

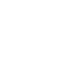

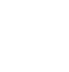
更

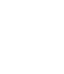

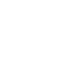

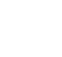

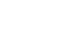

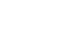

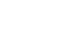

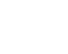

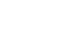

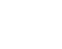


Membrane entropic contributions' hierarchy: unsaturation > tail length > curvature Membrane curvature effects are less relevant as the composition complexity grows Saturated Fatty Acids cause a higher stabilization on less organized membranes Unsaturated Fatty Acids cause a higher destabilization on more organized membranes 
Graphical Abștract.

a Stearic Acid (SA)

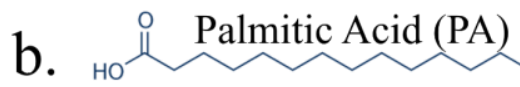

c. $\mathrm{H}_{\mathrm{N}}$ Oleic Acid (OA)

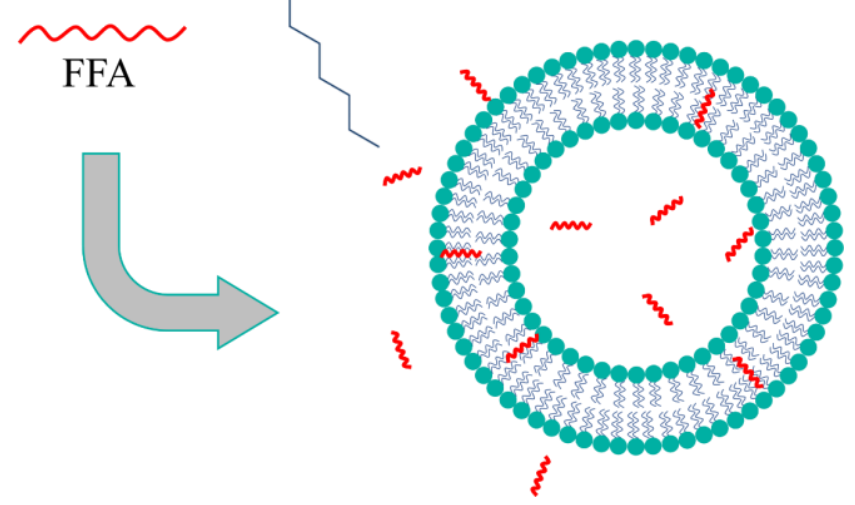

$\square$ Saturated ternary membrane

$\square$ Saturated ternary membrane $+5 \%$ DOPC

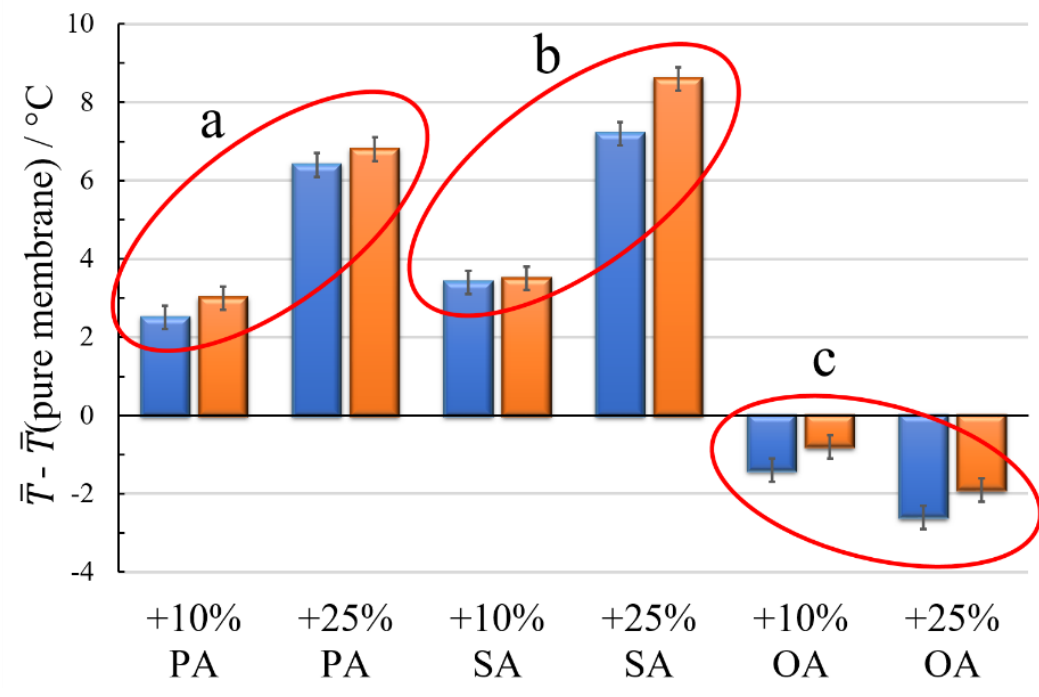

